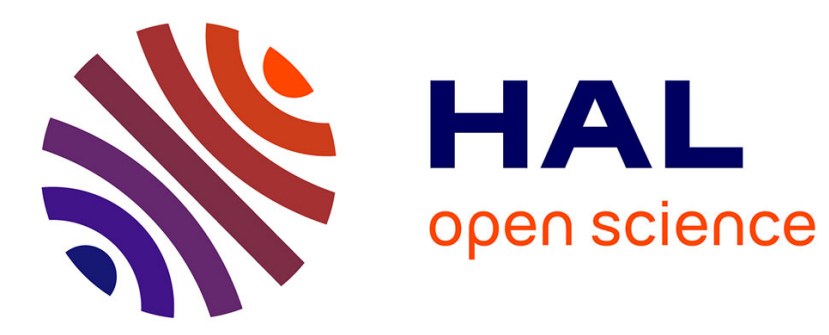

\title{
Finite element formulation for inflatable beams.
}

Anh Le Van, Christian Wielgosz

\section{To cite this version:}

Anh Le Van, Christian Wielgosz. Finite element formulation for inflatable beams.. Thin-Walled Structures, 2007, 45 (2), pp.221-236. 10.1016/j.tws.2007.01.015 . hal-01006899

\section{HAL Id: hal-01006899 \\ https://hal.science/hal-01006899}

Submitted on 6 Oct 2016

HAL is a multi-disciplinary open access archive for the deposit and dissemination of scientific research documents, whether they are published or not. The documents may come from teaching and research institutions in France or abroad, or from public or private research centers.
L'archive ouverte pluridisciplinaire HAL, est destinée au dépôt et à la diffusion de documents scientifiques de niveau recherche, publiés ou non, émanant des établissements d'enseignement et de recherche français ou étrangers, des laboratoires publics ou privés.

\section{(c)(1)}

Distributed under a Creative Commons Attribution| 4.0 International License 


\title{
Finite element formulation for inflatable beams
}

\author{
Anh Le van, Christian Wielgosz \\ GeM-Institute of Research in Civil and Mechanical Engineering, Faculty of Sciences in Nantes, 2, rue de la Houssiniere, \\ BP 92208, Nantes 44322 Cedex 3, France
}

The discretized nonlinear equations for bending and buckling of inflatable beams are written by use of the virtual work principle with Timoshenko's kinematics, finite rotations and small strains. The linearized equations around a pre-stressed reference configuration are then deduced, giving rise to a new inflatable beam finite element. The stiffness matrix contains the shear coefficient and the internal pressure. Use is made of the particular 3-node beam element to investigate the bending and the buckling of a cantilever beam, the deflection of a pinched torus and the buckling of a torus submitted to a radial compressive force. The numerical results obtained with the beam element are shown to be close to analytical and three-dimensional (3D) membrane finite element results. The validity of the numerical results is discussed, in connection with the concepts of the crushing force or the wrinkling pressure of the inflated beam.

Keywords: Inflatable beam; Thin-walled structure; Membrane structure; Follower force; Finite element

\section{Introduction}

Inflatable beam structures have several interesting properties: they are lightweight and easily folded. The internal pressure yields tensile pre-stressing in the fabrics and implies a significant bearing capacity, especially when the pressure is high. First analytical studies on inflatable beams are not new. Comer and Levy [1] studied an inflatable cantilever beam using the usual Euler-Bernoulli kinematics. Fichter [2] gave the first solution in which the internal pressure appears in the deflection expressions. Main et al. [3,4] made experiments on a cantilever and improved Comer's theory. More recently, Wielgosz and Thomas [5,6] have derived analytical solutions for inflated panels and tubes by using the Timoshenko kinematics and by writing the equilibrium equations in the deformed state of the beam in order to take into account the follower force effect of the internal pressure. They have obtained new expressions for the deflection and shown that the limit load is proportional to the applied pressure and that the deflections are inversely proportional to the material properties of the fabrics and to the applied pressure, thus improving Fichter's theory. Lately, Le van and Wielgosz [7] have used the virtual work principle with finite displacements and rotations in order to derive the nonlinear equations for inflatable beams; the linearized equations once again improve Fichter's theory.

Since analytical solutions are only viable for isostatic beams, finite elements are required for computing beam networks. Their development seems to be a recent topic of research; Wielgosz and Thomas [8] have constructed a finite element devoted to inflatable panels. This element including the inflation pressure effect was obtained by the equilibrium finite element method, according to which the global compliance is the sum of the yarn and beam compliances. This element has been converted in a displacement one in order to be easily used in usual finite element softwares. A finite element for tubes can also be found in [6], where the equilibrium method has once again been used, giving rise to a non-symmetrical stiffness matrix depending on the pressure, which is suitable for lightly ventilated structures. 
The aim of this paper is to derive a new displacement finite element for inflatable beams directly from the virtual work principle. The result will be a symmetrical stiffness matrix which holds for airtight tubes.

In the first section of the paper, the finite element is derived for the static in-plane stretching and bending problem of an inflated cylindrical beam made of a membrane. First, the discretized nonlinear equations will be written by use of the virtual work principle with Timoshenko's kinematics, finite rotations and small strains. The procedure will enable one to correctly account for the shear effect and the pressure in the governing equations. Then, the tangent stiffness matrix will be derived as the sum of a tangent matrix due to the internal strain work and another tangent matrix due to the internal pressure. Whereas it is essential to assume finite rotations in order to correctly exhibit all the terms due the internal pressure, it is sensible to use the inflatable beam in small deformations only. Thus, the nonlinear problem will be linearized around a well-defined reference configuration. This will lead to the inflatable beam finite element involving the Timoshenko shear coefficient and the inflation pressure. The particular case of a 3-node beam finite element with quadratic shape functions will be considered.

The 3-node beam finite element will be used in the next sections to deal with the bending and the buckling of a cantilever inflatable beam, the bending of a pinched toroidal beam and the buckling of a toroidal beam submitted to a radial compressive force. In every case, the finite element results will be compared with the analytical or 3D membrane finite element results, showing the good performance of the proposed finite element. Also, the validity of the numerical results will be discussed, in connection with the concepts of the crushing force or the wrinkling pressure of the inflated beam.

The formulation and results presented in this paper are the finite element counterpart of Ref. [7].

\section{The finite element for an inflatable beam}

Consider an inflatable beam made of a cylindrical membrane undergoing axial stretch and bending in the $x y$-plane (Fig. 1) under the combined action of an internal pressure and other external dead loads. In the reference (or initial) configuration, the length of the beam is $L_{o}$, the cross-section area $S_{0}$, the second moment of area $I_{0}$, and all the centroids $G_{o}$ of the cross-sections lie on the $x$-axis. The reference thickness of the membrane is $h_{o}$.

Remark. For inflatable structures, it should be emphasized that the loading is applied in two successive stages: first, the beam is inflated to pressure $p$, and then the other external forces are applied. At the beginning of the first stage, the internal pressure is zero and the beam is in a natural (or stress free) state. On the other hand, the reference configuration, which corresponds to the end of the first stage (before external forces other than the internal pressure are applied), is in a prestressed state. To clearly distinguish between the two states, we shall use index $\varnothing$ to denote the quantities in the natural state, as opposed to the usual index o for quantities related to the reference configuration. Thus, $L_{\varnothing}$ and $h_{\varnothing}$ designate the natural length and thickness; while $L_{o}$ and $h_{o}$ the reference ones. Computing $L_{o}$ and $h_{o}$ corresponding to a given pressure may be difficult depending on the problem at hand, but this is an independent subject which actually is common to every problem with a pre-stressed reference configuration.

In order to derive the discretized equations for the inflatable beam, use will be made of the principle of virtual work in the three-dimensional (3D) Lagrangian form

$$
\begin{aligned}
& \forall \text { virtual displacement field } \mathbf{V}^{*}, \\
& \quad-\int_{\Omega_{0}} \Pi^{\mathrm{T}}: \operatorname{grad} \mathbf{V}^{*} \mathrm{~d} \Omega_{0}+\int_{\Omega_{0}} \mathbf{f}_{o} \cdot \mathbf{V}^{*} \mathrm{~d} \Omega_{0}+\int_{\partial \Omega_{0}} \mathbf{V}^{*} \cdot \boldsymbol{\Pi} \cdot \mathbf{N} \mathrm{d} S_{0}=0,
\end{aligned}
$$

where $\Omega_{0}$ is the 3D region occupied by the beam in the reference configuration, $\partial \Omega_{0}$ its boundary, $\boldsymbol{\Pi}=\mathbf{F} \boldsymbol{\Sigma}$ and $\boldsymbol{\Sigma}$ the first (non-symmetric) and second (symmetric) Piola-Kirchhoff stress tensors ( $\mathbf{F}$ is the deformation gradient), $\mathbf{f}_{o}$ the body force per unit reference volume and $\mathbf{N}$ the unit outward normal in the reference contribution. Following the Timoshenko beam model, the displacement of any material point $\mathbf{P}_{\mathbf{o}}(X, Y, Z)$ in the beam is given by:

$$
\mathbf{U}\left(\mathbf{P}_{\mathbf{0}}\right)=\mathbf{U}+\mathbf{R}(\theta) \cdot \mathbf{G}_{\mathbf{0}} \mathbf{P}_{\mathbf{0}},
$$

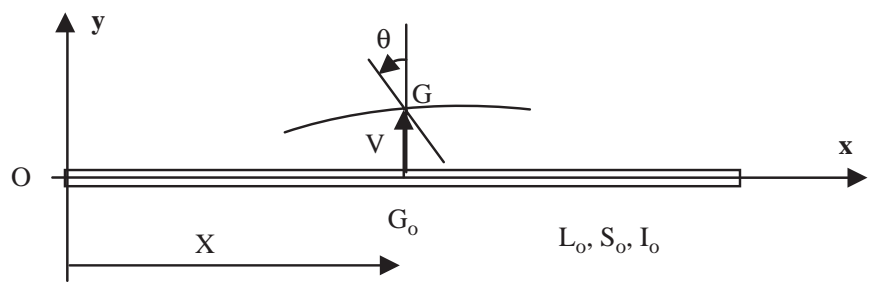

Fig. 1. The inflated beam model. 
where $\mathbf{U}(X)=(U(X), V(X), 0)$ is the displacement of the centroid of a current cross-section at abscissa $X$ (all the components are related to the base $(\mathbf{x}, \mathbf{y}, \mathbf{z})$, Fig. 1), $\theta(X)$ the finite rotation of the cross-section and $\mathbf{R}(\theta)$ the rotation tensor. The virtual displacement of a current material point $\mathbf{P}_{\mathbf{o}}$ is chosen as

$$
\mathbf{V}^{*}\left(\mathbf{P}_{\mathbf{o}}\right)=\mathbf{V}^{*}+\mathbf{\Omega}^{*} \times \mathbf{G P},
$$

where $\mathbf{V}^{*}(X)=\left(U^{*}(X), V^{*}(X), 0\right)$ is the virtual displacement of the centroid of a current cross-section, $\mathbf{\Omega}^{*}(X)=\left(0,0, \theta^{*}(X)\right)$ is the virtual rotation. Note that relation (3) involves the current position $\mathbf{G P}$, not the initial position $\mathbf{G}_{\mathbf{0}} \mathbf{P}_{\mathbf{o}}$, like the real velocity field in rigid body mechanics. The material is assumed to be hyperelastic and isotropic, obeying the Saint-Venant Kirchhoff constitutive law. The axial and shear components of the second Piola-Kirchhoff stress are then related to the components of the Green strain tensor by

$$
\Sigma_{X X}=\Sigma_{X X}^{0}+E \cdot E_{X X} \quad \Sigma_{X X}=\Sigma_{X Y}^{0}+2 G \cdot E_{X Y} \quad\left(\mathbf{G}=\frac{E}{2(1+v)}\right),
$$

where $E$ is the Young modulus, $v$ the Poisson ratio and $\Sigma_{X X}^{0}, \Sigma_{X Y}^{0}$ are the initial stresses induced by the preliminary inflation of the beam.

\subsection{Discretization of the internal virtual work}

As shown in [7], the internal virtual work in relation (1) can be recast as

$$
\begin{aligned}
\int_{\Omega_{0}} \boldsymbol{\Pi}^{\mathrm{T}}: \operatorname{grad} V^{*} \mathrm{~d} \Omega_{0}= & \int_{0}^{L_{o}}\left(\left[N\left(1+U_{, X}\right)+M \cos \theta \theta_{, X}-T \sin \theta\right] U_{, X}^{*}+\left[\left(N V_{, X}+M \sin \theta \theta_{, X}+T \cos \theta\right)\right] V_{, X}^{*}\right. \\
& +\left[M\left(-\left(1+U_{, X}\right) \sin \theta \theta_{, X}+V_{, X} \cos \theta \theta_{, X}\right)-T\left(\left(1+U_{, X}\right) \cos \theta+V_{, X} \sin \theta\right)\right] \theta^{*} \\
& +\left[M\left(\left(1+U_{, X}\right) \cos \theta+V_{, X} \sin \theta\right)+\int_{S_{0}} Y^{2} \Sigma_{, X X} \mathrm{~d} \mathbf{S}_{0} \theta_{, X}\right] \theta_{, X}^{*} \mathrm{~d} X
\end{aligned}
$$

where the generalized stresses - the (material) axial force, the shear force and the bending moment acting on the reference cross-section $S_{0}$-are given by

$$
\begin{aligned}
N & =\int_{S_{0}} \Sigma_{X X} \mathrm{~d} S_{0}=N^{o}+E S_{0}\left(U_{, X}+\frac{1}{2} U_{, X}^{2}+\frac{1}{2} V_{, X}^{2}+\frac{I_{o}}{2 S_{0}} \theta_{, X}^{2}\right) \\
T & =\int_{S_{0}} \Sigma_{X Y} \mathrm{~d} S_{0}=T^{o}+G S_{0}\left(V_{, X} \cos \theta-\left(1+U_{, X}\right) \sin \theta\right), \\
M & =-\int_{S_{0}} Y \Sigma_{X X} \mathrm{~d} S_{0}=M^{o}+E I_{0}\left(\left(1+U_{, X}\right) \cos \theta+V_{, X} \sin \theta\right) \theta_{, X}
\end{aligned}
$$

In relations (6), $N^{o}, T^{o}$ and $M^{o}$ are the resultants of the initial stresses on the cross-section. In practice, the coefficient $G S_{0}$ in (6b) is replaced by $\mathrm{kGS} S_{0}$, where the so-called correction shear coefficient $k$ is determined from the shape of the crosssection. The value usually found in the literature (see, e.g., [9]) for circular thin tubes is $k=0.5$. By assuming that the initial stress $\Sigma_{X X}^{0}$ takes the following form general enough in practical purposes: $\Sigma_{X X}^{0}=\alpha^{0}+\beta^{0} Y+\gamma^{0} Y^{2}$ and that $\Sigma_{Y Y}^{0}$ does not depend on $Y$, it was shown in [7] that the integral $\int_{S_{0}} Y^{2} \Sigma_{, X X} \mathrm{~d} S_{0}$ in (5) is

$$
\int_{S_{0}} Y^{2} \Sigma_{, X X} \mathrm{~d} S_{0}=\frac{N I_{0}}{S_{0}}+\frac{1}{2} K E \theta_{, X}^{2}+K \gamma^{o}
$$

where $K=\int_{S_{0}} Y^{4} \mathrm{~d} S_{0}-I_{0}^{2} / S_{0}$ is a quantity depending on the initial geometry of the cross-section, like $S_{0}$ and $I_{0}$. This quantity is involved in the nonlinear theory only, not in the linearized theory, as will be seen later. In order to discretize expression (5), one considers the beam as a finite element of length $L_{0}$, and interpolates the displacements and the rotation by

$$
U=\left\langle N_{U}\right\rangle\{U\}^{e} \quad V=\left\langle N_{V}\right\rangle\{V\}^{e} \quad \theta=\left\langle N_{\theta}\right\rangle\{\theta\}^{e},
$$

where the brackets \langle\rangle designate row-vectors and the braces \{\} column-vectors. The row-vectors $\left\langle N_{U}\right\rangle,\left\langle N_{V}\right\rangle,\left\langle N_{\theta}\right\rangle$ are the shape functions for the displacements $(U, V)$ and the rotation $\theta$. The column-vectors $\{U\}^{e},\{V\}^{e}$ and $\{\theta\}^{e}$ contain the nodal displacements and rotations (the superscript $e$ stands for 'element'). The virtual quantities $U^{*}, V^{*}, \theta^{*}$ are 
interpolated in the same way. The virtual internal work in the element writes

$$
\int_{\Omega_{o}} \boldsymbol{\Pi}^{\mathrm{T}}: \operatorname{grad} \mathbf{V}^{*} \mathrm{~d} \Omega_{0}=\int_{0}^{L_{o}}\left\langle\begin{array}{llll}
U_{, X}^{*} & V_{, X}^{*} & \theta^{*} & \left.\theta_{, X}^{*}\right\rangle \\
C \\
D
\end{array}\right\} \mathrm{d} X
$$

where

$$
\left\{\begin{array}{l}
A \\
B \\
C \\
D
\end{array}\right\}=\left\{\begin{array}{l}
N\left(1+U_{, X}\right)+M \cos \theta \theta_{, X}-T \sin \theta \\
N V_{, X}+M \sin \theta \theta_{, X}+T \cos \theta \\
-M\left(1+U_{, X}\right) \sin \theta \theta_{, X}+M V_{, X} \cos \theta \theta_{, X}-T\left[\left(1+U_{, X}\right) \cos \theta+V_{, X} \sin \theta\right] \\
M\left(1+U_{, X}\right) \cos \theta+M V_{, X} \sin \theta+\frac{N I_{0}}{S_{0}} \theta_{, X}+\frac{1}{2} E K \theta_{, X}^{3}+K \gamma^{o} \theta_{, X}
\end{array}\right\}
$$

and

$$
\left\{\begin{array}{c}
U_{, x}^{*} \\
V_{, x}^{*} \\
\theta^{*} \\
\theta_{, x}^{*}
\end{array}\right\}=[G]\left\{\begin{array}{l}
U^{*} \\
V^{*} \\
\theta^{*}
\end{array}\right\}=[G]\left\{\mathbf{U}^{*}\right\}^{e},[G]=\left[\begin{array}{lll}
\left\langle N_{U, x}\right\rangle & & \\
& \left\langle N_{V, x}\right\rangle & \\
& & \left\langle N_{\theta}\right\rangle \\
& \left\langle N_{\theta, x}\right\rangle
\end{array}\right] .
$$

The column-vector $\left\{\mathbf{U}^{*}\right\}^{e}$ gathers all the degrees of freedom $\left(U^{*}, V^{*}, \theta^{*}\right)$ of the element. Eventually, the virtual internal work is discretized as

$$
\int_{\Omega_{0}} \boldsymbol{\Pi}^{\mathrm{T}}: \operatorname{grad} \mathbf{V}^{*} \mathrm{~d} \Omega_{0}=\left\langle\mathbf{U}^{*}\right\rangle^{e}\{\boldsymbol{\Psi}\}^{e}
$$

with

$$
\{\boldsymbol{\Psi}\}^{e}=\int_{0}^{L_{o}}[G]^{\mathrm{T}}\left\{\begin{array}{l}
A \\
B \\
C \\
D
\end{array}\right\} \mathrm{d} X
$$

denoting the vector of element internal force.

\subsection{Discretization of the external virtual work due to the internal pressure}

Before external dead loads are applied, the beam is inflated by an internal pressure $p \cdot \mathbf{n}$ (where $\mathbf{n}$ is the outward unit normal in the current configuration), which gives the beam its bearing capacity. The contribution of the internal pressure is determined under the assumption that the reference volume $\Omega_{0}$ is a circular cylinder of radius $R_{o}$ and thickness $h_{o} \ll R_{o}$. This assumption, combined with the Timoshenko kinematics (2), implies that any cross-section remains a circular disk of radius $R_{o}$ during the deformation. This means that the change in shape (ovalization or warping) of the cross-section is not taken into account, as is the case of all usual beam models. The following expression for the virtual work of the pressure is obtained in [7]

$$
\begin{aligned}
W_{\text {pressure }}^{*}= & P \int_{0}^{L_{o}}\left[U^{*} \sin \theta \theta_{, X}-V^{*} \cos \theta \theta_{, X}+\theta^{*}\left(\cos \theta V_{, X}-\sin \theta\left(1+U_{, X}\right)\right)\right] \mathrm{d} X \\
& +P\left[U^{*} \cos \theta+V^{*} \sin \theta\right]_{0}^{L_{o}},
\end{aligned}
$$

where $P=p \pi R_{o}^{2}$. It should be mentioned that expression (14) is obtained since the current vector GP is involved in the virtual displacement (3) instead of the initial one $\mathbf{G}_{\mathbf{0}} \mathbf{P}_{\mathbf{0}}$. In order to get a symmetrical expression for the tangent stiffness 
matrix, let us recast the bracketed terms []$_{0}^{L_{o}}$ in relation (14) as follows:

$$
\begin{gathered}
{\left[U^{*} \cos \theta\right]_{0}^{L_{o}}=\int_{0}^{L_{o}}\left(U^{*} \cos \theta\right)_{, X} \mathrm{~d} X=\int_{0}^{L_{o}}\left(U_{, X}^{*} \cos \theta-U^{*} \sin \theta \theta_{, X}\right) \mathrm{d} X,} \\
{\left[V^{*} \sin \theta\right]_{0}^{L_{o}}=\int_{0}^{L_{o}}\left(V^{*} \sin \theta\right)_{, X} \mathrm{~d} X=\int_{0}^{L_{o}}\left(V_{, X}^{*} \sin \theta+V^{*} \cos \theta \theta_{, X}\right) \mathrm{d} X .}
\end{gathered}
$$

Hence,

$$
W_{\text {pressure }}^{*}=P \int_{0}^{L_{o}}\left[U_{, X}^{*} \cos \theta+V_{, X}^{*} \sin \theta+\theta^{*}\left(V_{, X} \cos \theta-\sin \theta-U_{, X} \sin \theta\right)\right] \mathrm{d} X .
$$

Eventually, relation (14) is discretized as

$$
W_{\text {pressure }}^{*}=\left\langle\mathbf{U}^{*}\right\rangle^{e}\left\{\boldsymbol{\Phi}_{\text {pressure }}\left(\mathbf{U}^{e}\right)\right\}^{e},
$$

where

$$
\left\{\boldsymbol{\Phi}_{\text {pressure }}\left(\mathbf{U}^{e}\right)\right\}^{e}=P \int_{0}^{L_{o}}[G]^{\mathrm{T}}\left\{\begin{array}{c}
\cos \theta \\
\sin \theta \\
V_{, X} \cos \theta-\sin \theta-U_{, X} \sin \theta \\
0
\end{array}\right\} \mathrm{d} X .
$$

\subsection{Tangent stiffness matrix}

The element tangent stiffness matrix is defined by

$$
[K]^{e}=\frac{\partial\{\boldsymbol{\Psi}\}^{e}}{\partial\{\mathbf{U}\}^{e}}-\frac{\partial\left\{\boldsymbol{\Phi}_{\text {pressure }}\right\}^{e}}{\partial\{U\}^{e}},
$$

where vectors $\{\boldsymbol{\Psi}\}^{e}$ and $\left\{\boldsymbol{\Phi}_{\text {pressure }}\right\}^{e}$ are given by (13) and (18). The first matrix in the right-hand side of (19) is standard:

$$
\frac{\partial\{\boldsymbol{\Psi}\}^{e}}{\partial\{\mathbf{U}\}^{e}}=\int_{0}^{L_{o}}[G]^{\mathrm{T}}\left([S]+[T]\left[\begin{array}{lll}
E S_{0} & & \\
& E I_{0} & \\
& & \mathrm{kGS} S_{0}
\end{array}\right][T]^{\mathrm{T}}\right)[G] \mathrm{d} X,
$$

where the symmetrical square matrix $[S]$ and the rectangular matrix $[T]$ are

$$
\begin{aligned}
& {[S]=\left[\begin{array}{cccc}
N & 0 & -M \sin \theta \theta_{, X}-T \cos \theta & M \cos \theta \\
& N & M \cos \theta \theta_{, X}-T \sin \theta & M \sin \theta \\
& & -M\left[\left(1+U_{, X}\right) \cos \theta+V_{, X} \sin \theta\right] \theta_{, X} & \\
& & +T\left[\left(1+U_{, X}\right) \sin \theta-V_{, X} \cos \theta\right] & -M\left[\left(1+U_{, X}\right) \sin \theta+V_{, X} \cos \theta\right] \\
\text { symmetrical } & & & N I_{0} \\
& & & S_{0}+\frac{3}{2} E K \theta_{, X}^{2}+K \gamma^{0}
\end{array}\right] .} \\
& {[T]=\left[\begin{array}{lll}
1+U_{, X} & \cos \theta \theta_{, X} & -\sin \theta \\
V_{, X} & \sin \theta \theta_{, X} & \cos \theta \\
0 & {\left[-\left(1+U_{, X}\right) \sin \theta+V_{, X} \cos \theta\right] \theta_{, X}} & -\left(1+U_{, X}\right) \cos \theta-V_{, X} \sin \theta \\
\frac{I_{0}}{S_{0}} \theta_{, X} & \left(1+U_{, X}\right) \cos \theta+V_{, X} \sin \theta & 0
\end{array}\right] .}
\end{aligned}
$$

The second matrix in the right-hand side of (19) is the element stiffness matrix due to the internal pressure and is essential for inflatable structures. After algebraic manipulations, one gets

$$
\frac{\partial\left\{\Phi_{\text {pressure }}\right\}^{e}}{\partial\{U\}^{e}}=\int_{o_{e}}^{L_{o}}[G]^{\mathrm{T}}\left[\begin{array}{llll}
0 & 0 & P \sin \theta & 0 \\
0 & 0 & -P \cos \theta & 0 \\
P \sin \theta & -P \cos \theta & P\left(V_{, X} \sin \theta+\cos \theta+U_{, X} \cos \theta\right) & 0 \\
0 & 0 & 0 & 0
\end{array}\right][G] \mathrm{d} X,
$$


which is a symmetrical matrix. Eventually, the global stiffness matrix is symmetrical and contains the material and the geometric matrices, including the presence of the internal pressure.

\subsection{General stiffness matrix for the linearized problem}

In engineering practice, the inflatable beam should be used in small deformations only. In this subsection, the nonlinear equations obtained above are linearized in order to get the stiffness matrix at the equilibrium state. It is noteworthy that it is crucial to derive the nonlinear equations first, assuming finite displacements and rotations, as done in the previous section, and then to carry out the linearization. Otherwise, if the linearization were carried out too early, some of the terms due the internal pressure would be missing. The linearization of the discretized equations will be performed about the reference configuration, which is in a pre-stressed state. For instance, in the case of bending, the pre-stress is due to the preliminary inflation of the beam; in the case of buckling, the pre-stress also includes the compressive load. The following assumptions are made on the initial stresses:

(i) The initial axial stress $\Sigma_{X X}^{0}$ is constant over the cross-section. Thus, $M^{o}$ in (6c) and $\gamma^{o}$ in (7) are zero.

(ii) The initial shear stress $\Sigma_{X Y}^{0}$ is zero. Thus, $T^{o}$ in (6b) is zero, too.

By taking these assumptions into account and making $U(X)=V(X)=\theta(X)=0$ in the tangent stiffness matrices (20)-(22), taking the definition (11) for matrix $[G]$ into account, one gets the element stiffness matrix for the linearized problem:

$$
[K]^{e}=\int_{o}^{L_{o}}\left[\begin{array}{lll}
\left(E S_{0}+N^{o}\right)\left\{N_{U, X}\right\}\left\langle N_{U, X}\right\rangle & & \\
& \left(k G S_{0}+N^{o}\right)\left\{N_{V, X}\right\}\left\langle N_{V, X}\right\rangle & -\left(k G S_{0}+P\right)\left\{N_{V, X}\right\}\left\langle\frac{N_{\theta}}{L_{o}}\right\rangle \\
& -\left(k G S_{0}+P\right)\left\{\frac{N_{\theta}}{L_{o}}\right\}\left\langle N_{V, X}\right\rangle & \frac{\left(E+N^{o} / S_{0}\right) I_{0}}{L_{o}^{2}}\left\{N_{\theta, X}\right\}\left\langle N_{\theta, X}\right\rangle+\left(k G S_{0}+P\right)\left\{\frac{N_{\theta}}{L_{o}}\right\}\left\langle\frac{N_{\theta}}{L_{o}}\right\rangle
\end{array}\right] \mathrm{d} X .
$$

\subsection{Particular case of the 3-node beam finite element}

All the subsequent numerical computations will be carried out using a 3-node element, with 3 degrees of freedom at each node (axial displacement $U$, deflection $V$ and rotation $\theta$ ). The three degrees of freedom are interpolated by the same quadratic shape function:

$$
\langle N\rangle=\left\langle\frac{1}{2} \xi(\xi-1) 1-\xi^{2} \frac{1}{2} \xi(\xi+1)\right\rangle \quad \xi \in[-1,+1] .
$$

Evaluating (23) with two integration points, at $\xi= \pm \frac{1}{\sqrt{3}}$, leads to the stiffness matrix for a 3 -node beam element of length $L_{o}$, as shown in expression (25). It should be noted that the two point integration corresponds to a reduced integration of the term $\left\{N_{\theta}\right\}\left\langle N_{\theta}\right\rangle$ and the full integration of the other terms. In the classical beam theory, $P$ is zero and the reduced integration provides exact solution when $\mathrm{kG} S_{o}$ tends to infinity:

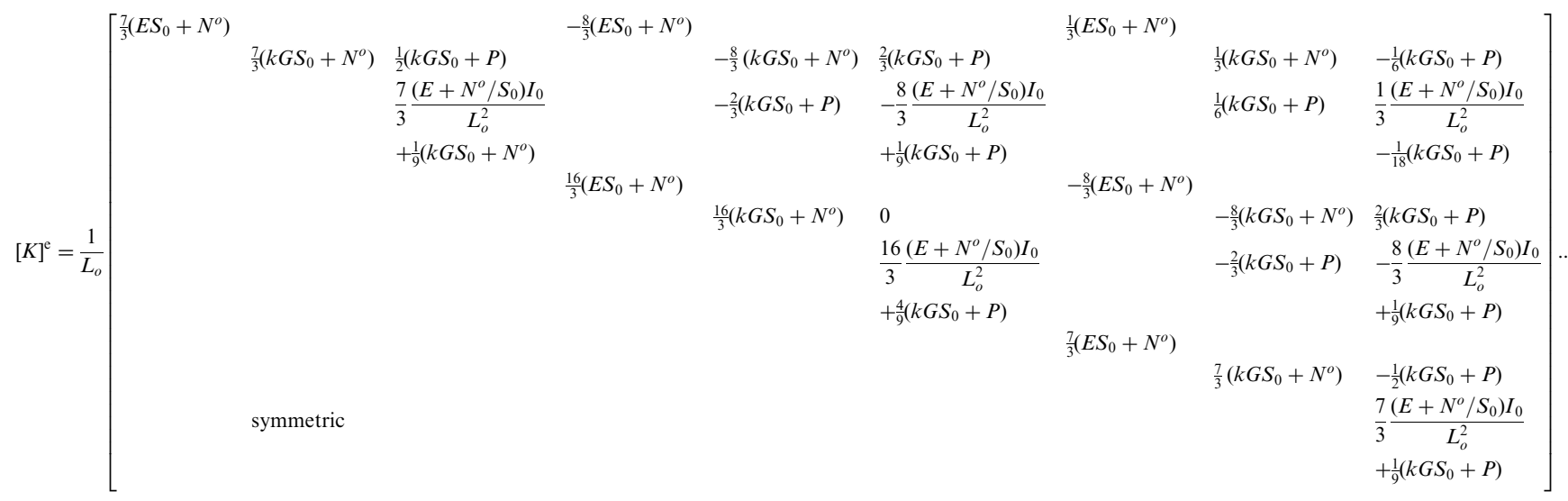




\section{Example 1. Bending of an inflatable cantilever beam}

Consider the linearized problem of an inflated cantilever beam under bending. The beam is made of a cylindrical membrane, its reference length is $\ell_{o}$, its reference radius $R_{o}$ and its reference thickness $h_{o}$. The beam is built-in at end $X=0$, subjected to an internal pressure $p$ and $a$ external force $F \mathbf{y}$ at end $X=\ell_{0}$ (see Fig. 4 and replace there the compressive force by the transverse load $F \mathbf{y}$ ).

\subsection{Comparison with analytical results}

Let us solve the problem using one single 3-node finite element (25). Here, the initial axial force is $N^{o}=P$. Since the axial displacement $U$ is zero, we will confine ourselves to the degrees of freedom $(V, \theta)$. Taking into account the boundary conditions $V_{1}=\theta_{1}=0$ yields the nodal deflections and rotations

$$
\begin{aligned}
V_{2} & =\frac{5 F \ell_{o}^{3}}{48\left(E+P / S_{0}\right) I_{0}}+\frac{F \ell_{o}}{2\left(k G S_{0}+P\right)}, \quad V_{3}=\frac{F \ell_{o}^{3}}{3\left(E+P / S_{0}\right) I_{0}}+\frac{F \ell_{o}}{k G S_{0}+P}, \\
\theta_{2} & =\frac{3 F \ell_{o}^{2}}{8\left(E+P / S_{0}\right) I_{0}}, \quad \theta_{3}=\frac{F \ell_{o}^{2}}{2\left(E+P / S_{0}\right) I_{0}} .
\end{aligned}
$$

Relations (26) coincide with the analytical solution proposed in [7]. The deflections and rotations are nonlinear functions of the pressure in two ways: First, the pressure is involved in the denominators through $P$; second, the reference dimensions $\ell_{o}, S_{0}$ and $I_{0}$ themselves depend on the pressure, as mentioned in Section 2.

Relations (26) are similar to those obtained by Fichter [2], apart from the additional term $P / S_{0}=p \pi R_{o}^{2} /\left(2 \pi R_{o} h_{o}\right)=$ $p R_{o} /\left(2 h_{o}\right)$. In some cases, this term may cause noticeable differences between Fichter's theory and the inflatable beam model proposed here. For instance, assume that $p=2 \times 10^{5} \mathrm{~Pa}, R_{o}=0.1 \mathrm{~m}, h_{o}=50 \times 10^{-6} \mathrm{~m}$ and $E=2.5 \times 10^{9} \mathrm{~Pa}$, then the term $P / S_{o}$ in (26) is equal to $8 \%$ of the Young's modulus $E$ and is thus not negligible compared to $E$.

If the internal pressure is zero, then $P=0$ and (26) coincides with the nodal values derived from the well-known analytical solution for a Timoshenko beam. However, contrary to a classical beam, here the inflatable beam is made of a membrane, so the pressure cannot be equal to zero for the beam not to collapse. This fact will be discussed below in connection with the validity of the solution. The influence of the internal pressure on the beam response is clearly shown in the previous relations: the inflation amounts to strengthen the Young's modulus and the shear modulus. In particular, when pressure $p$ tends to infinity, so do the equivalent material properties and the deflection and the rotation tend to zero.

\subsection{Limit of validity of the results}

Of course, one has to check a posteriori that the obtained deflection and rotation satisfy the small deformation hypothesis required by the linerization process. Yet there is another condition for the solution to hold: since the inflatable beam is made of a membrane, the principal stresses at any point in the beam must be non-negative. This amounts to satisfying the following inequality (see, e.g., $[1,10-13]$ for more details):

$$
F \leqslant \frac{\pi R_{o}^{3} p}{2 \ell_{o}}
$$

Recall that the reference length $\ell_{o}$ and radius $R_{o}$ are (increasing) functions of the pressure $p$. Inequality (27) shows that, given a force $F$, the internal pressure must be high enough for the bending solution to be meaningful. There exists a lower bound for the pressure, referred to as the wrinkling pressure of the beam, below which a wrinkle appears first at the point $\left(X=0, Y=R_{o}, Z=0\right)$ and the bending solution is no longer valid. For a given force $F$, Relation (27) is a rather intricate nonlinear inequality with the wrinkling pressure $p$ as unknown. A first-order approximation can be obtained by making in this inequality $\ell_{o} \approx \ell_{\varnothing}$ and $R_{o} \approx R_{\varnothing}$, which leads to the following simple bound where the right-hand side is known:

$$
p \geqslant p_{\text {wrinkling }} \equiv \frac{2 F \ell_{\varnothing}}{\pi R_{\varnothing}^{3}} .
$$

Table 1 gives the wrinkling pressures for $F=1 \mathrm{~N}$ and for a set of typical values for natural radius $R_{\varnothing}$ and natural length $\ell_{\varnothing}$.

Eventually, note that the moment at which the wrinkling occurs, which can be obtained from equality (28), $M_{\text {wrinkling }} \approx F \ell_{\varnothing} \leqslant\left(\pi R_{\varnothing}^{3} p / 2\right)$, is about 1.5 times less than the collapse moment given in [5], namely $M_{\text {collapse }}=$ $(\pi / 2)\left(\pi R_{\varnothing}^{3} p / 2\right)$ (at the collapse state, an increase in deflection does not result in an increase in moment) For a survey of various expressions for the collapse bending moment, see [14]. 
Table 1

Wrinkling pressure $p_{\text {wrinkling }}\left(\mathrm{N} / \mathrm{m}^{2}\right)$ given by relation (28) with $F=1 \mathrm{~N}$

\begin{tabular}{llll}
\hline Natural radius, $R_{\varnothing}(\mathrm{m})$ & Natural length $\ell_{\varnothing}(\mathrm{m})$ & & \\
\hline & 0.65 & 0.90 & 1.15 \\
0.04 & 6466 & 8952 & 11439 \\
0.06 & 1916 & 2653 & 3389 \\
0.08 & 808 & 1119 & 1430 \\
\hline
\end{tabular}

Table 2

Data for the inflatable cantilever beam

Natural thickness, $h_{\varnothing}$

Young modulus, $E$

Poisson ratio, $v$

Correction shear coefficient, $k$

Natural radius, $R_{\varnothing}(\mathrm{m})$

Natural length, $\ell_{\varnothing}(\mathrm{m})$

Pressure, $p\left(\mathrm{~N} / \mathrm{m}^{2}\right)$

0.04
0.65
$0.5 \times 10^{5}$

$125 \times 10^{-6} \mathrm{~m}$

$2.5 \times 10^{9} \mathrm{~N} / \mathrm{m}^{2}$

0.3

0.5

\section{Example 2. Buckling of an inflatable cantilever beam}

Consider now the same cantilever beam as in the previous section, and replace the bending force with an axial compressive force. As in the case of a classical beam, experiments show that, given an internal pressure, for low values of the compressive force $F$ there exists a unique solution corresponding to a uniaxial stress state where the beam remains straight. On the other hand, when force $F$ reaches some critical value, non-trivial solutions are possible, which correspond to a bent position of the beam. When computing the critical values by means of the finite element (25), one has to carry out numerical computations since it is impracticable to invert the stiffness matrix and obtain the closed-form expression for the critical force, even with one single element. The numerical results have been compared with the membrane finite element ones. Here, the initial axial force in relation (25) is $N^{o}=P-F$.

\subsection{Data for numerical computations}

The numerical computations are conducted with four values of the pressure $p$, three values of natural radius $R_{\varnothing}$ and three values of natural length $\ell_{\varnothing}$, while other quantities remain fixed, as shown in Table 2 .

As mentioned after relation (6), the correction shear coefficient $k$ is taken equal to the usual value for circular thin tubes, i.e. $k=0.5$. The reference dimensions $\left(\ell_{o}, R_{o}, h_{o}\right)$, defined as those when the beam is pressurized, are computed as functions of the internal pressure by means of the well-known elastic small strain solution for thin tubes:

$$
\begin{aligned}
& \ell_{0}=\ell_{\varnothing}\left(1+\frac{1-2 v}{2} \frac{p R_{\varnothing}}{E h_{\varnothing}}\right), \\
& R_{0}=R_{\varnothing}\left(1+\frac{2-v}{2} \frac{p R_{\varnothing}}{E h_{\varnothing}}\right), \\
& h_{o}=h_{\varnothing}-3 v \frac{p R_{\varnothing}}{2 E} .
\end{aligned}
$$

Recall that $\ell_{\varnothing}, R_{\varnothing}$ and $h_{\varnothing}$ are the natural length, radius and thickness, respectively. With the data in Table 2 , it is found that difference between the reference thickness and the natural thickness is $2.3 \%$ at most.

\subsection{Membrane finite element computations}

The numerical results obtained with the beam element will be compared with 3D membrane finite element results. The membrane computations are carried out using a general purpose nonlinear finite element program, based on the total Lagrangian formulation. The beam is modelled as a 3D membrane structure; the membrane elements have zero bending stiffness and satisfy the usual plane stress condition. The 3D constitutive law is the Saint-Venant Kirchhoff one, characterized by the Young's modulus $E$ and Poisson ratio $v$. The path-following and branch switching techniques are 


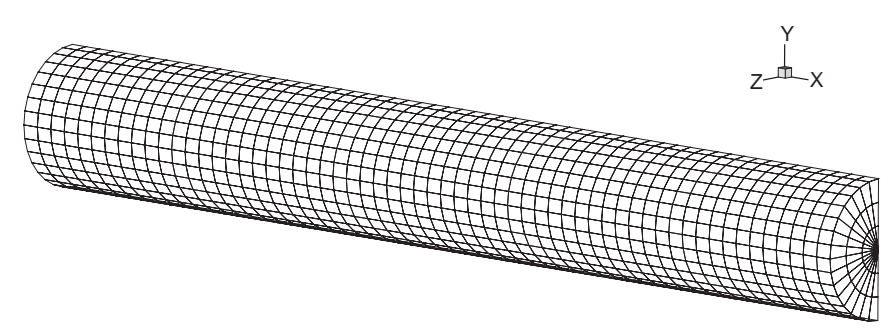

Fig. 2. A typical mesh used in 3D membrane finite element computations.

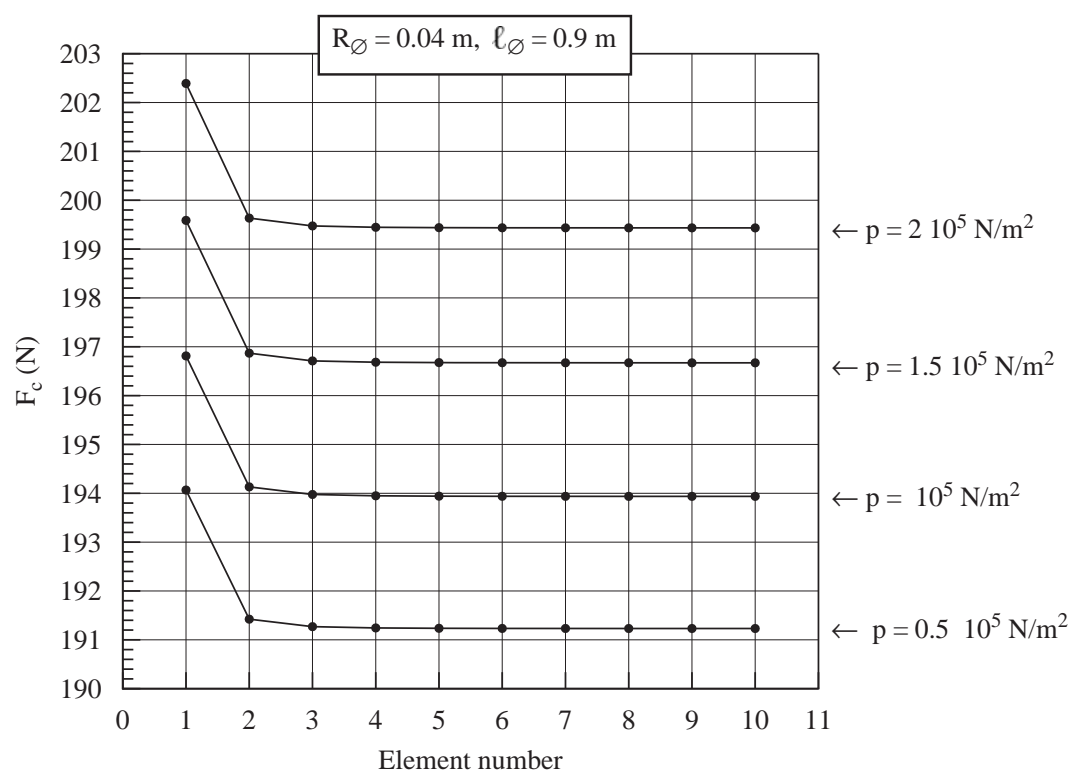

Fig. 3. Buckling of an inflatable cantilever beam. Mesh convergence test.

included in the numerical scheme, in order to deal with possible limit and bifurcation points. Fig. 2 shows a typical mesh used in 3D membrane finite element computations, containing 2401 nodes and 768 eight-node quadrilaterals and six-node triangles.

There is one significant difference between the inflatable beam model and the $3 \mathrm{D}$ membrane finite element solution. When solving by membrane finite element, the reference configuration is the natural one where the internal pressure is zero. On the other hand, when solving with the inflatable beam model the reference configuration is a pre-stressed state (after the pressure and the force $F$ are applied).

\subsection{Numerical results}

As shown in Fig. 3, the numerical results converge rapidly as the element number increases. Considering the geometry $R_{\varnothing}=0.04 \mathrm{~m}, \ell_{\varnothing}=0.9 \mathrm{~m}$ for instance, the critical force value $F_{c}$ obtained with more than 5 elements remains unchanged up to the fourth or even the fifth digit. Thus, the subsequent results are presented using 5 elements.

Fig. 4 gives the buckling load $F_{c}$ as a function of the length of the beam and the internal pressure. The values for $F_{c}$ obtained by the beam element are in good agreement with that obtained with the membrane finite element. Over the whole range of the computation, the differences are less than $0.9 \%$. The buckling force $F_{c}$ decreases as the tube length increases, and it increases along with the internal pressure. With $R_{\varnothing}=0.08 \mathrm{~m}$, for instance, $F_{c}$ is divided by about three from $\ell_{\varnothing}=0.65 \mathrm{~m}$ to $\ell_{\varnothing}=1.15 \mathrm{~m}$. With $R_{\varnothing}=0.08 \mathrm{~m}$ and $\ell_{\varnothing}=1.15 \mathrm{~m}, F_{c}$ rises by about $10 \%$ when the pressure varies from $0.5 \times 10^{5}$ to $2 \times 10^{5}$. Other computations, which are not presented here, show that the influence of the pressure on the buckling force is stronger if a material with lower Young's modulus is chosen. Nevertheless, the strains can then be so large that a fully nonlinear computation is required.

Note that the pressure plays a crucial role in the inflatable beam formulation: if the pressure were discarded in the stiffness matrix expression (25) (so that $N^{o}=-F$ and $P=0$ ), the discrepancy between the beam and the membrane finite element computations should reach $3.9 \%$. Concerning the role of the correction coefficient $k$, other numerical computations show that if $k$ is given very large values - so as to cancel the shear effect and switch from the Timoshenko to 

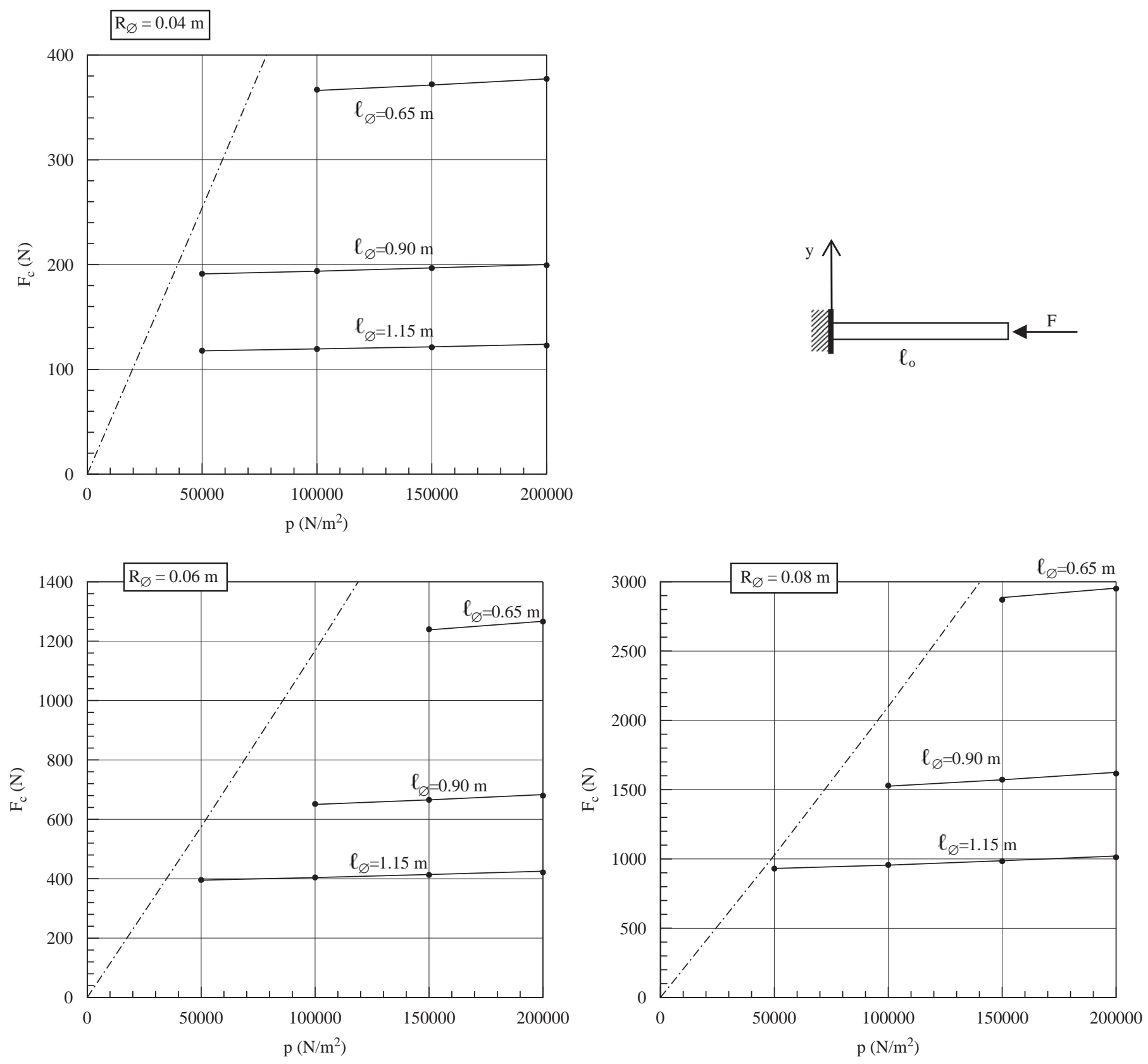

Fig. 4. Critical load of a cantilever. $\bullet$, inflatable beam finite element; relation (30).

the Euler-Bernoulli model - then the discrepancy reaches 8.7\%. In Ref. [7], the comparison was made between theoretical and membrane finite element results. The difference found therein are not the same, since the analytical and numerical results for the inflatable beam do not behave alike.

\subsection{Limit of validity of the numerical results}

Like the bending case, the computed buckling force is meaningful only if the internal pressure is high enough. Before buckling takes place, the principal stress directions at every point are directed along the cylindrical base vectors, so that the validity condition of the solution writes

$$
\Sigma_{X X}^{o}>0 \leftrightarrow N^{o}=P-F>0 \leftrightarrow F<F_{\text {crushing }} \equiv P=p \pi R_{o}^{2},
$$


where the reference radius $R_{o}$ itself is a function increasing with the pressure $p$. Inequality (30) shows that, given an internal pressure, the $F_{c}$ value obtained numerically must not be too high for the buckling solution to be meaningful. If the compressive force is greater than the upper bound specified by (30), the inflated beam collapses by crushing rather than by bending buckling. Thus, the bound given by (30) may be referred to as the crushing force of the inflated beam.

The crushing force is plotted versus the pressure in dashed lines in Fig. 4. Since reference radius $R_{o}$ is found to vary little with the pressure, these curves are almost straight lines. If the critical force $F_{c}$ is found lower than these curves, then the beam buckles at this value indeed. If not, the beam is crushed down before bending buckling occurs and the corresponding point $\left(p, F_{c}\right)$ is not represented in Fig. 4.

It is worth noting that the $3 \mathrm{D}$ membrane finite element computation does correctly detect the crushing forces as bifurcation points. However, it fails to determine the bifurcation eigenvector, which would mean that the beam is indeed in ultimate collapse. As expected, the plots of the critical force obtained by the 3D finite element computation versus the internal pressure stem from the crushing curves (dashed lines in Fig. 4). However, for the sake of clarity, these portions of curves are not represented.

\section{Example 3. Pinched toroidal beam}

Now consider a toroidal beam pressurized and compressed by two forces $F$ acting along a diameter (Fig. 5). The geometry is defined by the small radius $r_{\varnothing}$, the large radius $R_{\varnothing}$ and the membrane thickness $h_{\varnothing}$, which are natural quantities defined in the stress-free state before the internal pressure is applied.

The numerical computations are conducted on two geometries and four values of internal pressure $p$, using the data shown in Table 3.

The analytical solution for a torus subjected to an internal pressure was investigated by several authors. One of the most recent works is due to Redekop [15] who solved the problem in linear elasticity by means of successive approximations and obtained the analytical displacement field in the axisymmetrical case. The analytical solution for a non-axisymmetric pinched torus is not available to the authors' knowledge. Here, the toroidal beam will be computed by means of beam finite elements. The quarter of the beam (loaded by half of force $F$ ) is modeled with 20 three-node elements, which is more than
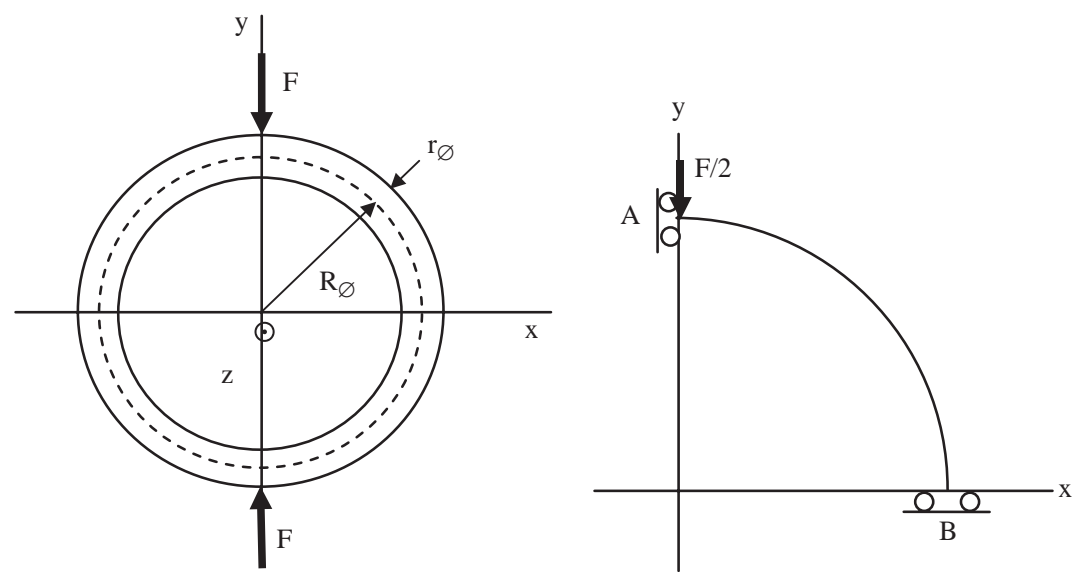

Fig. 5. Pinched toroidal beam and the modeled quarter.

Table 3

Data for the toroidal beam

Natural thickness, $h_{\varnothing}$

Young's modulus, $E$

The Poisson ratio, $v$

Correction shear coefficient, $k$

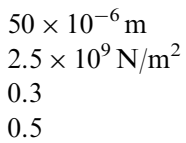

0.5

Geometry 1

Geometry 2

Natural small radius, $r_{\varnothing}(\mathrm{m})$

Natural large radius, $R_{\varnothing}(\mathrm{m})$

$\begin{array}{ll}0.01 & 0.02 \\ 1.00 & 1.00\end{array}$

Internal pressure, $p\left(\mathrm{~N} / \mathrm{m}^{2}\right)$

$0.5 \times 10^{5}$

$10^{5}$

$1.5 \times 10^{5}$

$2 \times 10^{5}$ 
enough (Fig. 6a). The numerical results will be compared with the 3D membrane finite element results. The membrane meshes contains 7817 nodes and $8 \times 300$ eight-node elements for geometry 1 , and 3917 nodes and $8 \times 150$ eight-node elements for geometry 2, Fig. 6(b).

When the beam is pressurized, it is assumed to remain toroidal, with small radius $r_{o}$ and large radius $R_{o}$. For lack of analytical expressions, these reference dimensions are taken equal to the values obtained by the $3 \mathrm{D}$ membrane finite element computations. Concerning the reference thickness $h_{o}$, it is approximated by relation (29c) for a straight beam. This is not accurate since, contrary to cylindrical beams studied above, here the pre-stresses in the reference state are not uniform over the beam section and so is the membrane thickness. Eventually, the equilibrium of half of the torus (without force $F$ ) shows that the initial axial force is $N^{o}=P$.

Fig. 7 shows a typical deformed shape of the torus. Table 4 and Fig. 8 give the displacements at points A and B of the torus (see Fig. 5) pinched by $F=10^{-2} \mathrm{~N}$. The displacements from the membrane computations can be obtained by two different manners, which result in identical values to within $6 \times 10^{-5}$ or less: for instance, the deflection $v_{B}$ is computed by

a

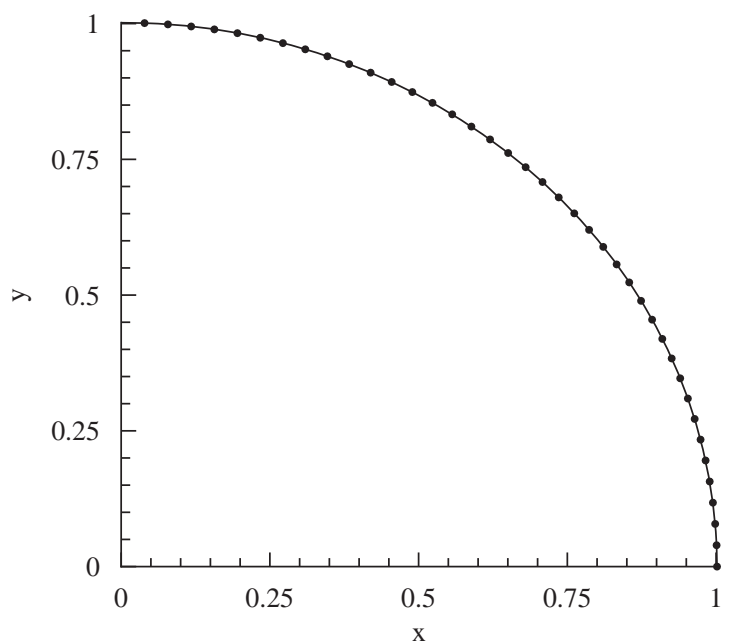

b

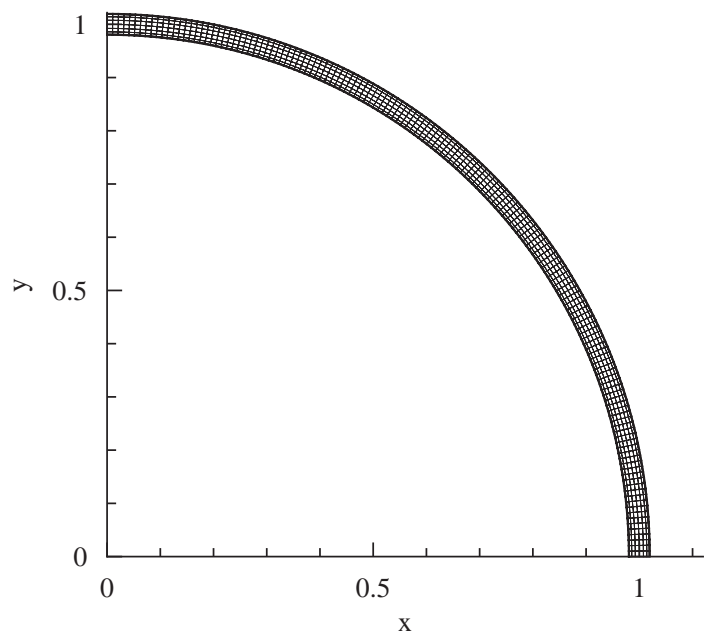

Fig. 6. Meshes of the pinched torus: (a) mesh with beam elements, (b) mesh for geometry 2, with membrane elements.

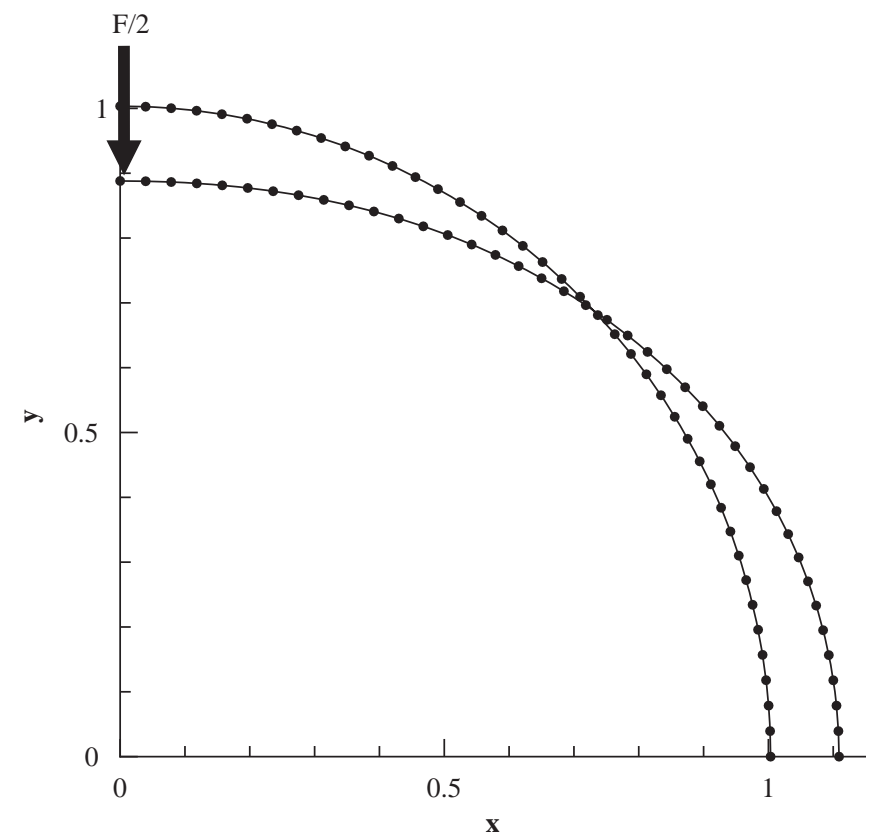

Fig. 7. Deformed shape of the pinched torus, $r_{\varnothing}=0.02 \mathrm{~m}, R_{\varnothing}=1 \mathrm{~m}, p=10^{5} \mathrm{~Pa}, F=10^{-2} \mathrm{~N}$. Magnifying factor for the displacement $=500$. 
Table 4

Displacements at points $\mathrm{A}$ and $\mathrm{B}$ of the torus pinched by $F=10^{-2} \mathrm{~N}$

\begin{tabular}{|c|c|c|c|}
\hline$p\left(\mathrm{~N} / \mathrm{m}^{2}\right)$ & Using beam element & Using 3D membrane element & Difference \\
\hline & $v_{A}(\mathrm{~m})$ & & \\
\hline $0.5 \times 10^{5}$ & $-0.1880 \mathrm{E}-02$ & $-0.1891 \mathrm{E}^{-} 02$ & $-0.6 \%$ \\
\hline $1.0 \times 10^{5}$ & $-0.1865 \mathrm{E}-02$ & $-0.1868 \mathrm{E}^{-} 02$ & $-0.1 \%$ \\
\hline $1.5 \times 10^{5}$ & $-0.1851 \mathrm{E}-02$ & $-0.1848 \mathrm{E}-02$ & $0.2 \%$ \\
\hline \multirow[t]{2}{*}{$2.0 \times 10^{5}$} & $-0.1837 \mathrm{E}-02$ & $-0.1828 \mathrm{E}-02$ & $0.5 \%$ \\
\hline & $u_{B}(\mathrm{~m})$ & & \\
\hline $0.5 \times 10^{5}$ & $0.1725 \mathrm{E}-02$ & $0.1732 \mathrm{E}-02$ & $-0.4 \%$ \\
\hline $1.0 \times 10^{5}$ & $0.1711 \mathrm{E}-02$ & $0.1711 \mathrm{E}-02$ & $0.0 \%$ \\
\hline $1.5 \times 10^{5}$ & $0.1698 \mathrm{E}-02$ & $0.1692 \mathrm{E}-02$ & $0.4 \%$ \\
\hline $2.010^{5}$ & $0.1686 \mathrm{E}-02$ & $0.1674 \mathrm{E}-02$ & $0.7 \%$ \\
\hline \multicolumn{4}{|c|}{ (a) Geometry 1: $R_{\varnothing}=1 \mathrm{~m}, r_{\varnothing}=0.01 \mathrm{~m}$} \\
\hline & $v_{A}(\mathrm{~m})$ & & \\
\hline $0.5 \times 10^{5}$ & $-0.2343 \mathrm{E}-03$ & $-0.2355 \mathrm{E}-03$ & $-0.5 \%$ \\
\hline $1.0 \times 10^{5}$ & $-0.2308 \mathrm{E}-03$ & $-0.2297 \mathrm{E}-03$ & $0.5 \%$ \\
\hline $1.5 \times 10^{5}$ & $-0.2274 \mathrm{E}-03$ & $-0.2248 \mathrm{E}-03$ & $1.1 \%$ \\
\hline $2.0 \times 10^{5}$ & $-0.2241 \mathrm{E}-03$ & $-0.2202 \mathrm{E}-03$ & $1.8 \%$ \\
\hline \multicolumn{4}{|c|}{ (b) Geometry 2: $R_{\varnothing}=1 \mathrm{~m}, r_{\varnothing}=0.02 \mathrm{~m}$} \\
\hline & $u_{B}(\mathrm{~m})$ & & \\
\hline $0.5 \times 10^{5}$ & $0.2145 \mathrm{E}-03$ & $0.2155 \mathrm{E}-03$ & $-0.5 \%$ \\
\hline $1.0 \times 10^{5}$ & $0.2112 \mathrm{E}-03$ & $0.2102 \mathrm{E}-03$ & $0.5 \%$ \\
\hline $1.5 \times 10^{5}$ & $0.2081 \mathrm{E}-03$ & $0.2057 \mathrm{E}-03$ & $1.1 \%$ \\
\hline $2.0 \times 10^{5}$ & $0.2051 \mathrm{E}-03$ & $0.2015 \mathrm{E}-03$ & $1.8 \%$ \\
\hline
\end{tabular}

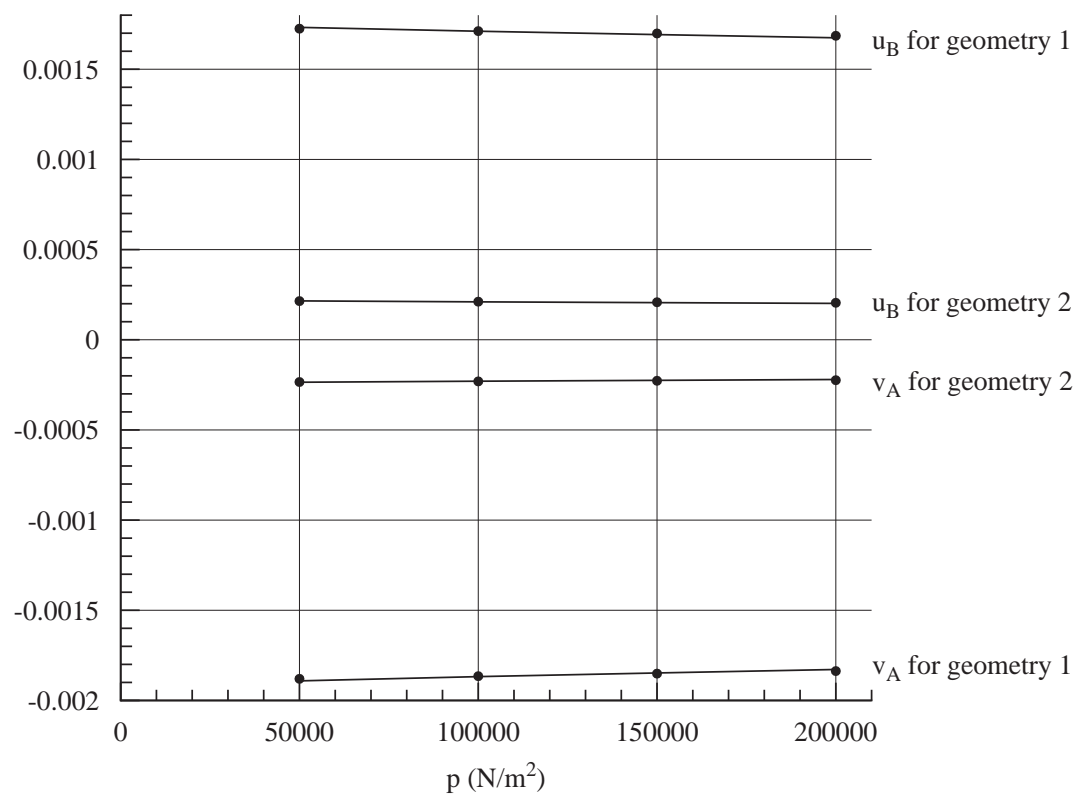

Fig. 8. Displacements (unit: m) at points A and B (Fig. 5) of the torus pinched by $F=10^{-2}$ N. $\bullet$, inflatable beam finite element; $\longrightarrow$, membrane finite element.

taking either the displacement at the point $\left(X=0, Y=R_{o}, Z=r_{o}\right)$, or the average of the displacements at the points $\left(X=0, Y=R_{o}+r_{o}, Z=0\right)$ and $\left(X=0, Y=R_{o}-r_{o}, Z=0\right)$. The results obtained by the inflatable beam element are in quite good agreement with those by the $3 \mathrm{D}$ membrane element, the maximum difference on $v_{A}$ or $u_{B}$ is $1.8 \%$.

Unlike other examples, a formula for the limit of validity for the pinched beam seems unavailable, because the loading is not axisymmetric and the stress state in the membrane due to the internal pressure and the pinching forces is rather intricate. The 3D membrane computations are the only direct way to know when a wrinkle appears and when the beam solution is no longer valid. It has been checked that the value $F=10^{-2} \mathrm{~N}$ chosen for the pinching force does not yield wrinkles. 


\section{Example 4. Buckling of an inflatable toroidal beam}

Consider now the same toroidal beam as in the previous section, and replace the pinching force $F$ with a radial compressive force $q$ per unit length uniformly distributed on the external circumference, Fig. 9. During the deformation process, the load $q$ is assumed to remain radial, i.e. directed toward the initial center of the ring. Let us compute the critical value of $q$, which produces some slight deflection from the circular form of equilibrium. We will deliberately preclude outof-plane buckling modes and confine ourselves to in-plane modes, although the former may occur before the latter.

\subsection{Numerical results}

As in the case of the pinched torus, only a quarter of the beam is modeled using 20 three-node elements, see Fig. 6(a). Table 5 and Fig. 10 show the buckling force as a function of the internal pressure, for the two considered geometries $\left(r_{\varnothing}=0.01 \mathrm{~m}, R_{\varnothing}=1 \mathrm{~m}\right.$ and $\left.r_{\varnothing}=0.02 \mathrm{~m}, R_{\varnothing}=1 \mathrm{~m}\right)$. The critical force $q_{c}$ increases as a quasi-linear function of the pressure. In addition, when the small radius $r_{\varnothing}$ is doubled, the critical force $q_{c}$ is multiplied by about 8 . The maximal difference with the $3 \mathrm{D}$ membrane finite element is $2.4 \%$.

The obtained results can also be compared with Weeks' analytical solution [16]. Weeks determined the buckling load $q_{c}$ of a pressurized toroidal ring subjected to a uniformly distributed radial load $q$, assuming linear elasticity and accounting for the prebuckling membrane stresses. The critical load is the root of the determinant of the global stiffness:

$$
\operatorname{det}\left(K^{\text {mat }}+q K^{g e o m}\right)=0,
$$

where $K^{\text {mat }}$ and $K^{\text {geom }}$ denote the material and the geometrical stiffness matrices, respectively. Eq. (31) leads to solving a cubic equation with unknown $q$. However, since the matrices in (31) are rather intricate, Weeks gave a first-order approximation to the critical load by expanding the determinant and keeping only the terms which are linear in $q$ and $r_{o} / R_{o}$. The result of this procedure is found to give answers within $5 \%$ of the exact solution for $r_{o} / R_{o} \leqslant 0.1$. Using our notations, it

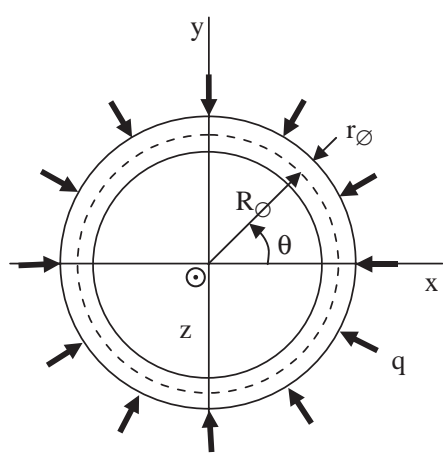

Fig. 9. Inflatable toroidal beam under radial compressive force.

Table 5

The critical force of a radially loaded inflatable toroidal beam

Pressure, $p\left(\mathrm{~N} / \mathrm{m}^{2}\right) \quad$ Reference small radius, $r_{o}(\mathrm{~m}) \quad$ Reference large radius, $R_{o}(\mathrm{~m}) \quad$ Critical force $q_{c}(\mathrm{~N} / \mathrm{m})$

Using beam element Using 3D membrane element Difference

(a) Geometry 1: $r_{\varnothing}=0.01 \mathrm{~m}, R_{\varnothing}=1 \mathrm{~m}$

$0.5 \times 10^{5} \quad 1.003 \mathrm{E}-02$

$1.0 \times 10^{5} \quad 1.007 \mathrm{E}-02$

$1.5 \times 10^{5} \quad 1.010 \mathrm{E}-02$

$2.0 \times 10^{5} \quad 1.013 \mathrm{E}-02$

$1.0008 \mathrm{E}+00$

$1.0016 \mathrm{E}+00$

$1.0025 \mathrm{E}+00$

$1.0034 \mathrm{E}+00$

(b) Geometry 2: $r_{\varnothing}=0.02 \mathrm{~m}, R_{\varnothing}=1 \mathrm{~m}$

$0.5 \times 10^{5} \quad 2.013 \mathrm{E}-02$

$1.0 \times 10^{5} \quad 2.027 \mathrm{E}-02$

$1.5 \times 10^{5} \quad 2.040 \mathrm{E}-02$

$2.0 \times 10^{5}$

$2.054 \mathrm{E}-02$

$\begin{array}{rrrr}1.0016 \mathrm{E}+00 & 1.246 \mathrm{E}+01 & 1.216 \mathrm{E}+01 & 2.4 \% \\ 1.0034 \mathrm{E}+00 & 1.265 \mathrm{E}+01 & 1.253 \mathrm{E}+01 & 1.0 \% \\ 1.0052 \mathrm{E}+00 & 1.284 \mathrm{E}+01 & 1.280 \mathrm{E}+01 & 0.3 \% \\ 1.0071 \mathrm{E}+00 & 1.302 \mathrm{E}+01 & 1.309 \mathrm{E}+01 & -0.5 \%\end{array}$

Comparison with 3D membrane element. 


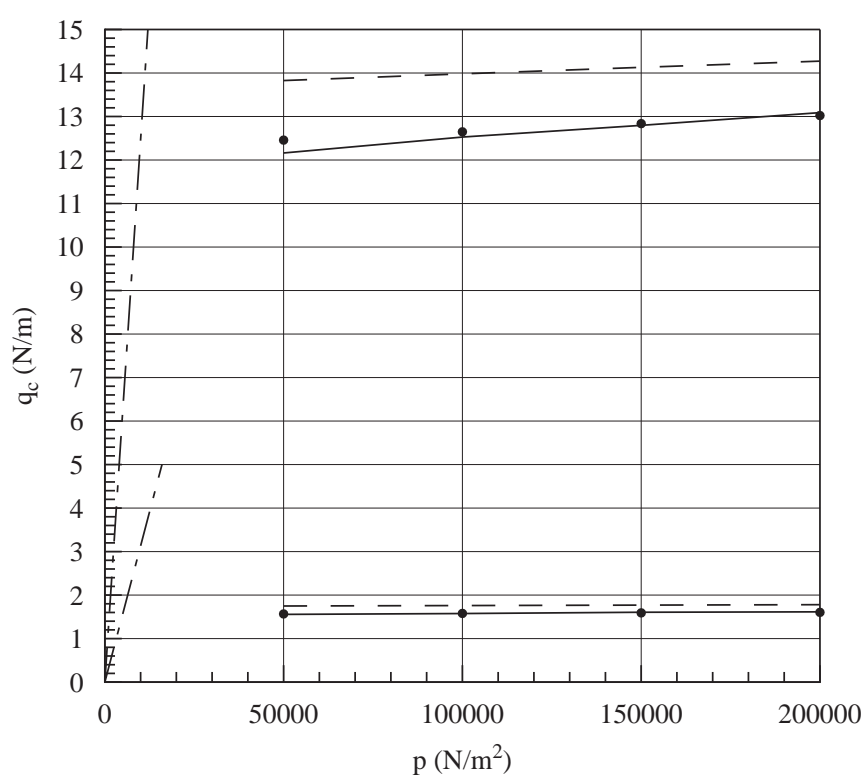

Fig. 10. The critical force of a radially loaded inflatable toroidal beam. $\bullet$, inflatable beam finite element; weeks' solution, relation (32); _ - _ _ : crushing force $q_{\text {crushing }}$ given by relation (33).

reads

$$
q_{\mathrm{cr}}=\frac{4.5 \frac{E I_{0}}{R_{o}^{3}}}{1+3 r_{o} / 2 R_{o}+\frac{\left(4+4.5 r_{o} / R_{o}\right) E I_{0} / R_{o}^{2}}{G S_{0} / 2+p \bar{S}_{0}}} \quad \text { with } \quad G=\frac{E}{2(1+v)}, \quad S_{o}=2 \pi r_{o} h_{o}, \quad \bar{S}_{o}=\pi r_{o}^{2}, \quad I_{0}=\pi r_{o}^{3} h_{o} .
$$

As Weeks did not tell how to compute the reference dimensions $r_{o}, R_{o}$ and $h_{o}$, these are obtained here from the 3D membrane finite element computations or approximated by relation $(29 \mathrm{c})$, as said above. The numerical values for $q_{c}$ from (32) are reported in Fig. 10 in dashed lines. For the ratios $r_{o} / R_{o}$ retained here, the differences between Weeks' results and ours are about $10 \%$, which could be considered as reasonable in view of the various approximations made.

\subsection{Limit of validity of the numerical results}

By denoting $\theta$ the circumferential direction of the large circle (see Fig. 9), the membrane solution is meaningful as long as the hoop stress $\Sigma_{\theta \theta}^{o}$ - or the initial axial force $N^{o}$-is positive. The expression for $N^{o}$ is obtained from the equilibrium of half the torus under the combined action of the internal pressure $p$ and the external load $q$ :

$$
\Sigma_{\theta \theta}^{o}>0 \leftrightarrow 2 N^{o}=2 P-2 q\left(R_{o}+r_{o}\right)>0 \leftrightarrow q<q_{\text {crushing }} \equiv \frac{P}{R_{o}+r_{o}}=\frac{p \pi r_{o}^{2}}{R_{o}+r_{o}},
$$

where the reference radii $R_{o}$ and $r_{o}$ vary as functions of the pressure $p$. If the compressive force $q$ is greater than the upper bound specified by (33), the inflated torus collapses so that the bound may be referred to as the crushing force of the inflated torus. Using results in Table 5, the crushing force is plotted versus the pressure in dash-dot lines in Fig. 10. As in the case of the bent cantilever beam, these curves are almost straight lines. For the two considered geometries, the critical forces $q_{c}$ obtained in Table 5 are acceptable since they are lower than the crushing forces.

\section{Conclusions}

Inflatable structures can be computed with 3D thin shell or membrane finite elements. However, a lot of structures can be analyzed with beam finite elements so as to significantly reduce the computing time. The main features of the paper are summarized below:

(i) A new beam finite element has been obtained, involving the Timoshenko shear coefficient and the inflation pressure. The explicit expression for the stiffness matrix has been derived in the particular case 3-node beam element. 
(ii) It is crucial to distinguish between the natural configuration where the internal pressure is zero and the pre-stressed reference configuration around which the linearization is performed. The dimensions defining the reference configuration of the beam depend on the prescribed internal pressure.

(iii) For the bending problem of a cantilever beam, one single element is able to provide the analytical solution. For the buckling of a cantilever beam, the bending and the buckling of a toroidal beam, the numerical results have compared well with the membrane finite element results. Over the whole range of the computations, the differences are lower than $2.4 \%$ compared with those given by the membrane analysis, showing the good performance of the proposed finite element.

(iv) The numerical results for the bent cantilever beam only holds if the pressure is greater than the so-called wrinkling pressure. Similarly, the solutions for the buckled cantilever beam and the toroidal beam are valid only if the compressive force is less than the so-called crushing force.

The proposed formulation applies to the static in-plane stretching and bending problem of inflated beams. Taking into account inertia effects is quite straightforward unless fluid interaction is significant (here the fluid is the inflated air). On the other hand, the equations for 3D problems should be rather lengthy. Further investigations are in progress in order to obtain finite elements for inflated beam structures in 3D space, in statics and dynamics.

\section{References}

[1] Comer RL, Levy S. Deflections of an inflated circular cylindrical cantilever beam. AIAA J 1963;1(7):1652-5.

[2] Fichter WB. A theory for inflated thin-wall cylindrical beams. NASA Technical Notes D-3466, 1966.

[3] Main A, Peterson SW, Strauss AM. Load-deflection behaviour of space-based inflatable fabric beams. J Aerospace Eng 1994;2(7):225-38.

[4] Main JA, Peterson SW, Strauss AM. Beam-type bending of space-based membrane structures. J Aerospace Eng 1995;8(2):120-5.

[5] Wielgosz C, Thomas JC. Deflections of inflatable fabric panels at high pressure. Thin-Walled Struct 2002;40:523-36.

[6] Thomas JC, Wielgosz C. Deflections of highly inflated fabric tubes. Thin-Walled Struct 2004;42:1049-66.

[7] Le van A, Wielgosz C. Bending and buckling of inflatable beams: some new theoretical results. Thin-Walled Struct 2005;43:1166-87. Erratum: Thin-Walled Struct 2006; 44: 822-23.

[8] Wielgosz C, Thomas JC. An inflatable fabric beam finite element. Commun Num Methods Eng 2003;19:307-12.

[9] Cowper GR. The shear coefficient in Timoshenko's beam theory. J Appl Mech 1967;33:335-40.

[10] Stein M, Hedgepeth JM. Analysis of partly wrinkled membranes. NASA Technical Note, NASA TN D-813, 1961. p. 1-23.

[11] Bulson PS. Design principles of pneumatic structures. Struct Eng 1973;51(6):209-15.

[12] Webber JPH. Deflections of inflated cylindrical cantilever beams subjected to bending and torsion. Aeronaut J 1982;86(10):306-12.

[13] Haughton DM, McKay BA. Wrinkling of inflated elastic cylindrical membranes under flexure. Int J Eng Sci 1996;34:1531-50.

[14] Veldman SL, Bergsma OK, Beukers A. Bending of anisotropic inflated cylindrical beams. Thin-Walled Struct 2005;43:461-75.

[15] Redekop D. A displacement solution in toroidal elasticity. Int J Vessels Piping 1992;51:189-209.

[16] Weeks GE. Buckling of a pressurized toroidal ring under uniform external loading. NASA Technical Note, NASA TN D-4124, August 1967. 